\title{
COVID-19 in patients undergoing long-term dialysis in Ontario
}

\author{
Leena Taji MPH, Doneal Thomas MSc, Matthew J. Oliver MD, Jane Ip BASc, Yiwen Tang MSc, Angie Yeung BSc MBA, \\ Rebecca Cooper BA LLB, Andrew A. House MD, Phil McFarlane MD, Peter G. Blake MSc MB
}

Cite as: CMAJ 2021 February 22;193:E278-84. doi: 10.1503/cmaj.202601; early-released February 4, 2021

CMAJ Podcasts: author interview at www.cmaj.ca/lookup/doi/10.1503/cmaj.202601/tab-related-content

\begin{abstract}
BACKGROUND: Patients undergoing longterm dialysis may be at higher risk of infection with severe acute respiratory syndrome coronavirus 2 (SARS-CoV-2) and of associated disease and mortality. We aimed to describe the incidence, risk factors and outcomes for infection in these patients in Ontario, Canada.
\end{abstract}

METHODS: We used linked data sets to compare disease characteristics and mortality between patients receiving longterm dialysis in Ontario who were diagnosed SARS-CoV-2 positive and those who did not acquire SARS-CoV-2 infection, between Mar. 12 and Aug. 20, 2020. We collected data on SARS-CoV-2 infection prospectively. We evaluated risk factors for infection and death using multivariable logistic regression analyses.

RESULTS: During the study period, 187 $(1.5 \%)$ of 12501 patients undergoing dialysis were diagnosed with SARS-CoV-2 infection. Of those with SARS-CoV-2 infection, 117 (62.6\%) were admitted to hospital and the case fatality rate was $28.3 \%$. Significant predictors of infection included in-centre hemodialysis versus home dialysis (odds ratio [OR] 2.54, 95\% confidence interval $[\mathrm{Cl}]$ 1.59-4.05), living in a long-term care residence (OR 7.67,
95\% Cl 5.30-11.11), living in the Greater Toronto Area (OR 3.27, 95\% Cl 2.21-4.80), Black ethnicity (OR 3.05, 95\% Cl 1.954.77), Indian subcontinent ethnicity (OR $1.70,95 \% \mathrm{Cl} 1.02-2.81)$, other non-White ethnicities (OR 2.03, 95\% Cl 1.38-2.97) and lower income quintiles (OR 1.82, 95\% Cl 1.15-2.89).

INTERPRETATION: Patients undergoing long-term dialysis are at increased risk of SARS-CoV-2 infection and death from coronavirus disease 2019. Special attention should be paid to addressing risk factors for infection, and these patients should be prioritized for vaccination.

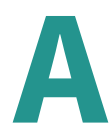
s of Aug. 20, 2020, in Ontario, Canada's most populous province, almost 41000 people had tested positive for severe acute respiratory syndrome coronavirus 2 (SARSCoV-2), the virus that causes coronavirus disease 2019 (COVID19), ${ }^{1}$ which represented $0.3 \%$ of the provincial population. Close to 2800 people had died, a case fatality rate of $6.8 \% .^{2}$

Patients undergoing dialysis have high rates of comorbid conditions, are often older adults, have varying degrees of immunosuppression and are more likely to reside in long-term care, which puts them at risk of both acquiring SARS-CoV-2 and developing complicated disease. ${ }^{3,4}$ Furthermore, in Ontario, those who receive in-centre hemodialysis typically have 3 treatments per week in outpatient units located in or affiliated with hospitals, and the consequent inability to fully selfisolate means that patients undergoing hemodialysis likely have an even higher risk of SARS-CoV-2 infection., ${ }^{3,4}$ Recent studies support this but do not compare infection rates with those in the local population of patients not undergoing dialysis..$^{5-10}$ Several studies have reported SARS-CoV-2 infection in single or multicentre cohorts of patients undergoing dialysis, ${ }^{5-10}$ but we are unaware of any that have identified risk factors for infection at the level of a large region. Some studies have found that patients with SARS-CoV- 2 infection who are undergoing dialysis are at high risk of severe illness and death. ${ }^{6-10}$

\section{Methods}

\section{Study setting}

Ontario has a population of about 14.5 million. More than 12000 people receive long-term dialysis, with about $74 \%$ receiving in-centre hemodialysis and $26 \%$ home dialysis, which comprises $21 \%$ peritoneal dialysis and 5\% home hemodialysis. All dialysis in Ontario is funded by a single payer - the provincial government - operating through the Ontario Renal Network (ORN), a provincial agency that funds and manages services for patients with chronic kidney disease within 27 Renal Programs, which provide home dialysis services and operate more than 100 in-centre hemodialysis units. ${ }^{11}$ The latter vary in size from 3 to 60 hemodialysis stations. To understand the impact of SARS-CoV-2 on 
patients who receive long-term dialysis, ORN developed a weekly surveillance data collection tool to gather key information on disease trajectory during the pandemic. Our objective was to describe the incidence, outcomes and risk factors for SARS-CoV-2 infection in this patient population and to measure outcomes including mortality.

Practices were changed over time in dialysis units in Ontario between March and August 2020, with increased availability of personal protective equipment, universal masking, easier access to SARS-CoV-2 testing and screening of patients before they entered hemodialysis units. It was common practice, especially in larger urban settings, for staff to work in more than 1 unit throughout this period.

\section{Study population}

We included all prevalent patients receiving long-term dialysis who were registered in the Ontario Renal Reporting System (ORRS) as of Mar. 31, 2020, and all new patients starting long-term dialysis who were registered in ORRS between Apr. 1 and Aug. 20, 2020. We defined patients who were undergoing long-term dialysis as those registered in a Renal Program and funded for nonacute dialysis, even if within 30 days of starting dialysis. We excluded patients from the analyses if their health card number, postal code, age or sex were missing from the ORRS, or if they were not residents of Ontario or younger than 18 years of age (Figure 1 ).

We defined patients with a diagnosis of SARS-CoV-2 infection as those who tested positive for the infection according to the Ontario government testing guideline, which states that laboratory confirmation of infection requires a validated assay, comprising a positive nucleic acid amplification test on at least 1 specific genome target. ${ }^{12}$ If patients received a negative result on repeat testing of the same or a subsequent swab in the same 24-hour period, we did not classify them as positive cases. Most testing was conducted in patients who were symptomatic or who had suspected exposure to people who were infected. A province-wide point prevalence testing study involving patients receiving hemodialysis carried out by ORN in June 2020 detected a minimal number of asymptomatic positive cases.

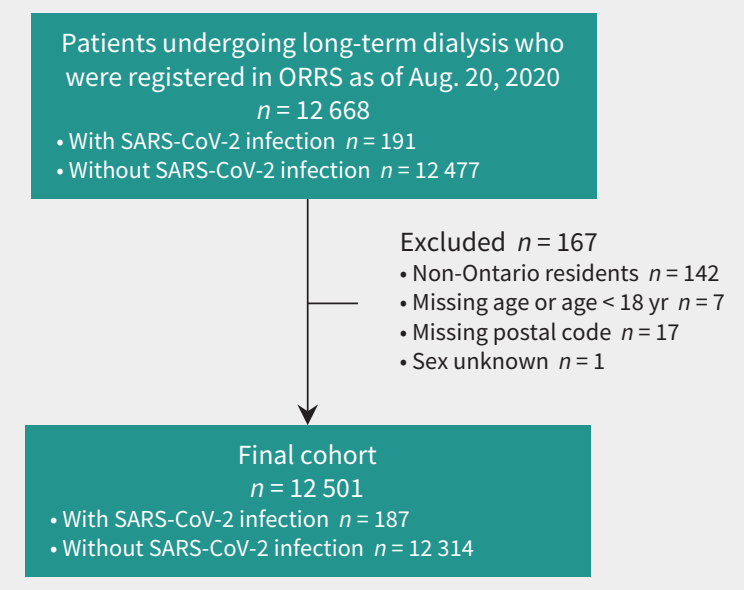

Figure 1: Flow chart for selection of the cohort of patients undergoing long-term dialysis in Ontario. Note: ORRS = Ontario Renal Reporting System, SARS-CoV-2 = severe acute respiratory syndrome coronavirus 2 .

\section{Data sources}

A manual data collection tool was developed to capture key information on patients with SARS-CoV-2 infection who were undergoing long-term dialysis in Ontario units: name, health card number, type of patient residence, name of institution, hospital admission status, dialysis modality, intensive care and ventilator status, and whether the patient had an active SARS-CoV-2 infection, was recovered or had died. We included deaths in the cohort up to 4 weeks after Aug. 20, 2020. We also collected personal health information that could be linked to other data sets.

Data were collected prospectively from the second week of March 2020, when the first patient with SARS-CoV-2 infection undergoing long-term dialysis was identified. On Apr. 9, 2020, all Renal Programs started submitting data on a weekly basis for all patients with SARS-CoV-2 infection. These weekly submissions included new cases and status updates for existing cases. Although not all of the patients were contacted each week, we considered the data complete because programs were in close, regular contact with all patients who were receiving in-centre and home dialysis. Data were collected and submitted by an experienced designated data lead at each program. Before the first data submission, orientation webinars were held to train all data leads on the data collection tool. We subsequently crosschecked data on hospital admissions with the Canadian Institute for Health Information Discharge Abstract Database (CIHI-DAD) and identified only 1 discrepant case.

We linked 7 data sets using unique, encoded patient identifiers, as well as postal codes. These were the ORN COVID-19 data collection tool; the ORRS, which identifies all patients receiving care for advanced kidney disease and dialysis in Ontario; the Registered Persons Database (RPDB), which provides demographic information for Ontario residents; the Postal Code Conversion File (Statistics Canada), which links postal codes to standard geographic areas; the Local Health Integration Network (LHIN) Sub-Regions Postal Code Crosswalk, which contains a mapping of postal codes to Ontario's health regions; $\mathrm{ClHI}$ DAD, which contains hospital admission diagnosis information for Ontario residents; and Ontario Health Insurance Plan (OHIP), which contains health insurance claims information for physician services.

We extracted demographic data on patient sex and age from the RPDB. Ethnicity information in the ORRS was collected by the data leads in each program at the time of patient registration, based on charting by clinical staff who could ask patients to self-identify ethnicity if necessary but were not mandated to do so. We linked postal codes to 2011 Statistic Canada Census data or LHIN Crosswalk to determine neighbourhood income quintiles or geographic location, respectively. We identified the latest dialysis modality from ORRS. We determined residency in long-term care from physicians' billing codes in the OHIP database that indicated long-term care services ("W" fee codes) in the 6-month period before the last service date in the fiscal year. We identified transplant history and comorbidities using the International Statistical Classification of Diseases and Related Health Problems 10th Revision codes in the OHIP or CIHI-DAD databases. We defined patients with cardiac disease as those with a history of myocardial infarction or congestive heart failure identified by relevant diagnostic codes on any hospital discharge (CIHI-DAD). Each chronic condition was defined based on 5 years of look-back data from the date of SARS-CoV-2 reporting. 


\section{Statistical analysis}

We compared the trend in new cases with that seen in the general Ontario population. We also compared characteristics of patients undergoing dialysis who were diagnosed with SARS-CoV-2 infection with those who tested negative, by analyzing data for the time period (Mar. 12 to Aug. 20, 2020). We calculated frequency (percentages) for categorical variables and means with standard deviations (SDs) or medians and interquartile ranges (IQRs) for continuous variables. We used standardized differences for univariate comparisons between patients with a diagnosis of SARSCoV-2 infection and those without infection. We considered a standardized difference greater than $10 \%$ to be meaningful. ${ }^{13}$

To explore risk factors associated with SARS-CoV-2 infection, we included all baseline variables in a multivariable logistic regression model. We used variance inflation factor to screen for multicollinearity. We considered values of 10 or higher to be strong evidence of multicollinearity; however, we did not find evidence of multicollinearity. We used SAS statistical software to perform the multivariable analysis and set statistical significance at 2-sided $p<0.05$.

\section{Analysis of additional data}

Data collection for patients undergoing dialysis in Ontario not included in the analysis already described continued subsequent to Aug. 20,2020. We calculated updated infection rates to include the period up to Jan. 21, 2021.

\section{Ethics approval}

The data collection was in accordance with Ontario Health's legislative authority under the Ontario Personal Health Information Protection Act, 2004.

\section{Results}

Peaks in trends of new cases for the general population and the population of patients undergoing dialysis coincided; although the peaks coincided, the decline of new cases among patients undergoing dialysis appeared faster compared with that in the general population (Figure 2). ${ }^{2}$ We found that the number of new infections diagnosed among patients undergoing long-term dialysis increased between Mar. 12, when the first case occurred, and Apr. 23, 2020, when the case rate peaked at 33 per week. Subsequently, this number steadily decreased. On Aug. 20, 2020, the 12-week average was just over 1 new case per week (Figure 2) and a total of 187 patients, $1.5 \%$ of the total dialysis population, had a diagnosis of SARS-CoV-2.

Almost $80 \%$ of cases among patients undergoing dialysis occurred in 8 of the 27 Renal Programs, and in 6 of these there was an "outbreak" (defined as 3 or more cases diagnosed in the same unit within a week). Seven programs had no cases.

Among patients undergoing dialysis, 187 were diagnosed with SARS-CoV-2 infection and 12314 tested negative. We did not classify as positive 7 cases in which the patient had discordant test results within a 24-hour period and the clinical judgment was that the positive test was false. The median age of those with infection was 68 years and $61.5 \%$ were male (Table 1 ). With regard to dialysis modality, $88.8 \%$ of infected patients were receiving in-centre hemodialysis compared with $74.4 \%$ of patients who did not have the infection (standardized difference $=40 \%$ ). Overall, $1.8 \%$ of the entire in-centre hemodialysis population in Ontario had a diagnosed infection compared with $0.3 \%$ of the patients undergoing home hemodialysis and $0.8 \%$ of the patients undergoing peritoneal dialysis.

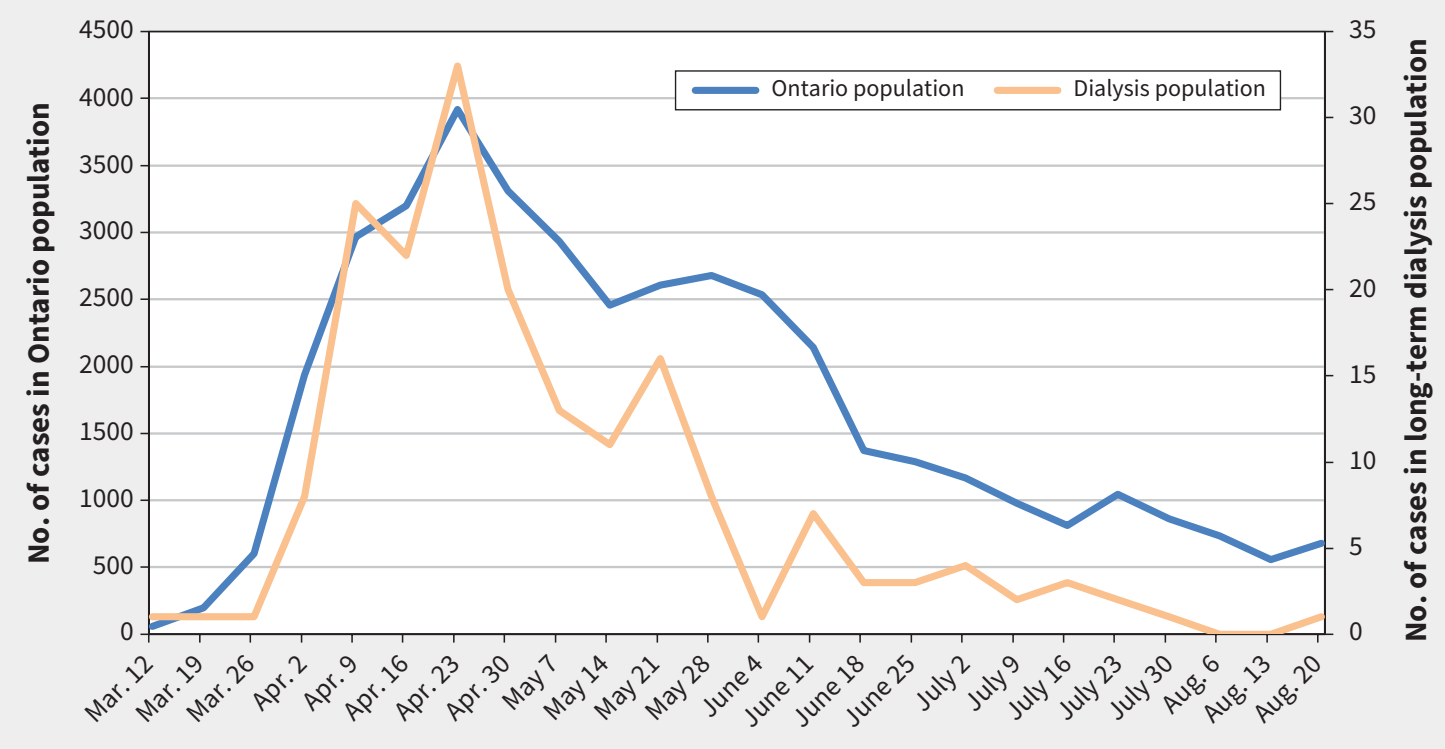

Date

Figure 2: Number of new cases of severe acute respiratory syndrome coronavirus 2 (SARS-CoV-2) infection among patients undergoing long-term dialysis in Ontario and the Ontario general population, ${ }^{2}$ per reporting week, as of Aug. 20, 2020. Note: We used 12501 as the denominator for the dialysis population; peak incidence was 33 cases $=264$ per 100000 population for the week ending Apr. 23, 2020. We used $14745040^{14}$ as the denominator for the general Ontario population for this period; peak incidence was 3918 cases $=27$ per 100000 population for the week ending Apr. 23,2020 . 
Table 1: Clinical and demographic characteristics of patients undergoing long-term dialysis in Ontario with and without a diagnosis of SARS-CoV-2 infection, as of Aug. 20, 2020

\begin{tabular}{|c|c|c|}
\hline Characteristic & $\begin{array}{l}\text { No. }(\%) \text { of patients with } \\
\text { SARS-CoV-2 infection }\end{array}$ & $\begin{array}{l}\text { No. }(\%) \text { of patients v } \\
\text { SARS-CoV-2 infec }\end{array}$ \\
\hline All patients & 187 (NA) & 12314 (NA) \\
\hline \multicolumn{3}{|l|}{ Modality } \\
\hline In-centre hemodialysis & $166(88.8)$ & $9159(74.4)$ \\
\hline Home dialysis & $21(11.2)$ & $3155(25.6)$ \\
\hline \multicolumn{3}{|l|}{ Years on dialysis } \\
\hline $0-5$ & $138(73.8)$ & $9754(79.2)$ \\
\hline$>5$ & $49(26.2)$ & $2560(20.8)$ \\
\hline \multicolumn{3}{|l|}{ Resident of LTC } \\
\hline No & $140(74.9)$ & $11753(95.4)$ \\
\hline Yes & $47(25.1)$ & $561(4.6)$ \\
\hline \multicolumn{3}{|l|}{ Geographic location } \\
\hline Greater Toronto Area & $151(80.7)$ & $6293(51.1)$ \\
\hline $\begin{array}{l}\text { Outside the Greater Toronto } \\
\text { Area }\end{array}$ & $36(19.3)$ & $6021(48.9)$ \\
\hline \multicolumn{3}{|l|}{ Sex } \\
\hline Female & $72(38.5)$ & 4909 (39.9) \\
\hline Male & $115(61.5)$ & $7405(60.1)$ \\
\hline \multicolumn{3}{|l|}{ Age group, yr } \\
\hline $18-49$ & $25(13.4)$ & $1654(13.4)$ \\
\hline $50-64$ & $57(30.5)$ & $3427(27.8)$ \\
\hline$\geq 65$ & $105(56.1)$ & $7233(58.7)$ \\
\hline \multicolumn{3}{|l|}{ Age, yr } \\
\hline Median (IQR), yr & $68(58-78)$ & $68(57-77)$ \\
\hline Range & 20-92 & $18-102$ \\
\hline \multicolumn{3}{|l|}{ Ethnicity } \\
\hline White & $61(32.6)$ & $7265(59.0)$ \\
\hline Black & $38(20.3)$ & $889(7.2)$ \\
\hline Indian subcontinent & $24(12.8)$ & $1177(9.6)$ \\
\hline Other non-White & $57(30.5)$ & $2675(21.7)$ \\
\hline Missing/unknown & $7(3.7)$ & $308(2.5)$ \\
\hline \multicolumn{3}{|l|}{ Diabetes } \\
\hline Yes & $86(46.0)$ & $5872(47.7)$ \\
\hline \multicolumn{3}{|l|}{ Cancer } \\
\hline Yes & $10(5.3)$ & $856(7.0)$ \\
\hline \multicolumn{3}{|l|}{ Cardiac disease } \\
\hline Yes & $53(28.3)$ & $3587(29.1)$ \\
\hline \multicolumn{3}{|l|}{ Previous transplant } \\
\hline Yes & $7(3.7)$ & $807(6.6)$ \\
\hline \multicolumn{3}{|l|}{ Income quintiles $\dagger$} \\
\hline 1 (lowest) & $69(36.9)$ & $3639(29.6)$ \\
\hline 2 & $49(26.2)$ & $2752(22.3)$ \\
\hline 3 & $42(22.5)$ & $2348(19.1)$ \\
\hline 4 & $15(8.0)$ & $1901(15.4)$ \\
\hline 5 (highest) & $12(6.4)$ & $1606(13.0)$ \\
\hline Missing/unknown & $0(0.0)$ & $68(0.6)$ \\
\hline
\end{tabular}

Note: IQR = interquartile range, LTC = long-term care, NA = not available, SARS-CoV-2 = severe acute respiratory syndrome coronavirus 2 .

*Accepted criteria for determining a meaningful difference is a standardized difference greater than $10 \%$ and is indicated in boldface type.

†Income quintile is a measure of neighbourhood socioeconomic status that divides the population into 5 income groups of equal size in which group 1 lives in the neighbourhoods with the lowest incomes and group 5 in those with the highest incomes. 
A larger proportion of patients who tested positive for SARSCoV-2 resided in long-term care; were of Black, Indian subcontinent and other non-White ethnicities; lived in the Greater Toronto Area; and were in the lowest 2 income quintiles (Table 1; Appendix 1, Supplementary Table 1, available at www.cmaj.ca/lookup/doi/10.1503/ cmaj.202601/tab-related-content).

\section{Predictors of SARS-CoV-2 infection and mortality}

The results of our multivariable analysis found the following to be independent predictors of SARS-CoV-2 infection among patients undergoing dialysis: in-centre hemodialysis; residence in long-term care; living in the Greater Toronto Area; Black, Indian subcontinent and other non-White ethnicities; and lower income quintiles. Age, diabetes and other comorbidities were not predictive (Figure 3; Appendix 1, Supplementary Table 1).

Of the 187 patients with SARS-CoV-2 infection, 53 (28.3\%) died and $117(62.6 \%)$ were admitted to hospital (37 [19.8\%] were admitted to ICU and 28 [15\%] received mechanical ventilation). During the same period, noninfected patients had a mortality rate of $5.8 \%$ and a hospital admission rate of $27 \%$. Among those patients with SARS-CoV-2 infection, our multivariable analysis showed that only older age was predictive of mortality.

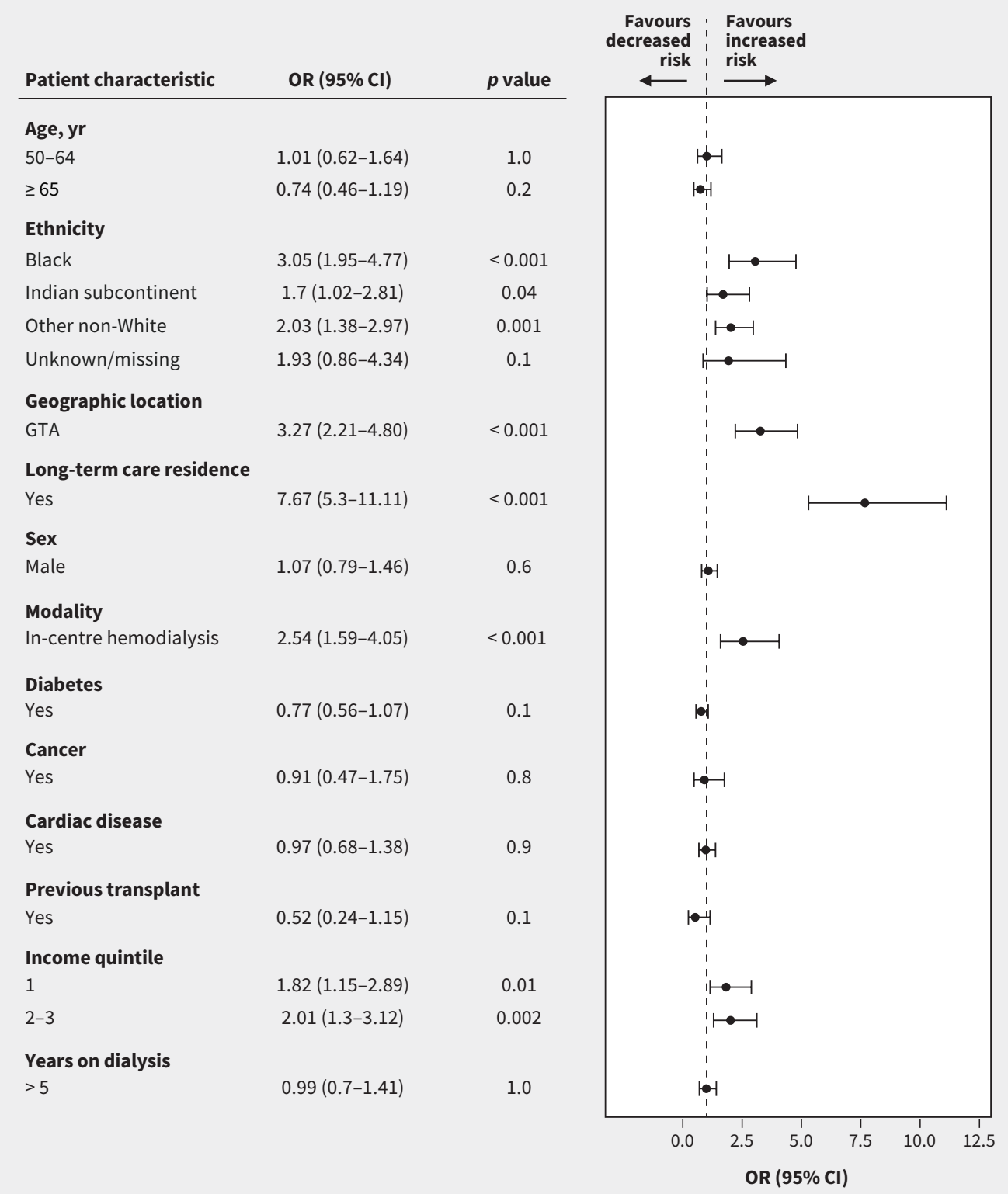

Figure 3: Summary of multivariable logistic regression predicting risk of severe acute respiratory syndrome coronavirus 2 (SARS-CoV-2) infection among patients undergoing long-term dialysis (no. of patients with a diagnosis of SARS-CoV-2 infection = 187; no. without SARS-COV-2 infection = 12246). Reference categories: age younger than 50 years, ethnicity White, geographic location outside the Greater Toronto Area (GTA), modality home dialysis, highest 2 income quintiles, 5 or fewer years undergoing dialysis, sex female and all other variables were No. Note: $\mathrm{Cl}=$ confidence interval, OR = odds ratio. We excluded 68 patients from the cohort without SARS-CoV-2 infection because linkage to neighbourhood income quintiles was not possible. 


\section{Additional data}

Between Aug. 21, 2020, and Jan. 21, 2021, another 386 patients receiving long-term dialysis were diagnosed with SARS-CoV-2 infection. In total, 573 patients were infected with SARS-CoV-2, which is $4.2 \%$ of the total dialysis population treated between Mar. 12, 2020, and Jan. 21, 2021, in Ontario.

\section{Interpretation}

In Ontario, 1.5\% of the population receiving long-term dialysis had a diagnosis of SARS-CoV-2 infection in the first 5 months of the 2020 pandemic. Of these patients, $62.6 \%$ were admitted to hospital, $19.8 \%$ required intensive care and $28.3 \%$ died. Rates of diagnosed infection were lower in patients on home dialysis compared with those on in-centre hemodialysis. Other risk factors for infection were living in the Greater Toronto Area; residence in long-term care; being of Black, Indian subcontinent and other non-White ethnicities; and lower income.

The rate of SARS-CoV-2 infection of $1.5 \%$ among patients undergoing dialysis in Ontario is lower than that seen in the United Kingdom, where more than $8 \%$ of patients undergoing dialysis were infected, ${ }^{15}$ but is higher than the $0.2 \%$ rate in British Columbia for the same time period (Dr. Adeera Levin, British Columbia Provincial Renal Association, personal communication, 2020). We have summarized incidence and mortality data for dialysis patients across 11 international jurisdictions in Appendix 2, Supplementary Table 2, available at www.cmaj.ca/lookup/

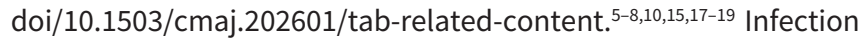
rates for SARS-CoV-2 in any population of people undergoing dialysis are likely influenced by those in the broader community. However, the $1.5 \%$ rate in Ontario's dialysis population is more than 5 times higher than that recorded for the general Ontario population during the same period. ${ }^{2}$ We found that residence in long-term care was a significant predictor of infection, with more than $25 \%$ of all cases occurring in this setting, consistent with findings from the general Ontario population and dialysis populations elsewhere. ${ }^{2,20,21}$ We also found that infection rates were 2.5 times higher among patients receiving in-centre hemodialysis than among those on home dialysis; a similar excess risk was reported in the UK. ${ }^{9,15,22}$ Patients who must visit dialysis units regularly are potentially exposed to infection, as suggested by our identification of multiple simultaneous cases in 6 centres.

Patients who were non-White comprised $67 \%$ of those with SARS-CoV-2 infection, whereas they comprise only $41 \%$ of all dialysis patients in Ontario. We also found a higher infection rate among patients identified as of Black or Indian subcontinent ethnicity, an association not noted in the UK. ${ }^{10}$ This finding was independent of diabetes and socioeconomic status and may reflect related factors such as high density, multigenerational living and well-recognized structural inequalities associated with health care disparities for these populations..$^{23,24}$

Our study has a number of strengths. We derived our data from a population of more than 14 million, with more than 12000 patients receiving long-term dialysis. The single-payer public funding and delivery of dialysis and acute care in hospital in Ontario enabled us to report reliably on the entire population of patients undergoing long-term dialysis. We began data collection at the start of the 2020 pandemic, and this prospective, standardized data collection allowed for detailed coverage of all diagnosed infections. Weekly reporting of cases allowed for accurate capture of incidence and outcomes over time.

\section{Limitations}

The higher infection rates among patients undergoing dialysis may be partly due to easier access to SARS-CoV-2 testing. Similarly, the higher rate for those undergoing in-centre hemodialysis compared with those undergoing home dialysis may be partly related to easier access to testing, given their regular in-person contact with medical staff and their participation in surveillance initiatives, even when asymptomatic. However, most SARS-CoV-2-positive patients were symptomatic. Surveillance testing was carried out in some hemodialysis units with outbreaks and once in June 2020 on a province-wide basis, but only a small number of asymptomatic positive cases were identified. Widespread serology testing was not sufficiently available or reliable to validate our results. Data were not available for some potential risk factors such as occupation, household density, respiratory disease and body weight.

\section{Conclusion}

Patients undergoing dialysis in Ontario who had a diagnosis of SARS-CoV-2 infection had a case fatality rate almost 4 times higher than that reported for the general Ontario population for the same period. Therefore, as the COVID-19 pandemic proceeds, focused efforts should be made to protect this population from infection including prioritizing patients on long-term dialysis and the staff treating them for SARS-CoV-2 vaccination. Apart from standard precautions, prevention strategies should include specific education of these patients about their risk of infection and associated high mortality. Paid sick leave for those patients who are required to work in risk-associated occupations should be provided. Other strategies should include a low symptom threshold for testing, increased space between treatment stations within hemodialysis units, universal droplet precautions, as well as regular surveillance testing of high-risk groups such as people living in long-term care. Encouragement of suitable patients to start home dialysis because of a lower infection risk has been reinforced and strategies to provide on-site dialysis in long-term care facilities are being developed.

\section{References}

1. Coronavirus disease 2019 (COVID-19). Toronto: Public Health Ontario. Available: www.publichealthontario.ca/en/diseases-and-conditions/infectious-diseases/ respiratory-diseases/novel-coronavirus (accessed 2020 July 13).

2. COVID-19: Epidemiologic summaries from Public Health Ontario. Toronto: Ontario Ministry of Health. Available: https://covid-19.ontario.ca/covid-19-epidemiologic -summaries-public-health-ontario (accessed 2020 Aug. 26).

3. Rombolà G, Brunini F. COVID-19 and dialysis: why we should be worried. J Nephrol 2020;33:401-3.

4. Meijers B, Messa P, Ronco C. Safeguarding the maintenance hemodialysis patient population during the coronavirus disease 19 pandemic. Blood Purif 2020;49:259-64.

5. Xiong F, Tang H, Liu L, et al. Clinical characteristics of and medical interventions for COVID-19 in hemodialysis patients in Wuhan, China. J Am Soc Nephrol 2020;31: 1387-97.

6. Valeri AM, Robbins-Juarez SY, Stevens JS, et al. Presentation and outcomes of patients with ESKD and COVID-19. J Am Soc Nephrol 2020;31:1409-15. 
7. Alberici F, Delbarba E, Manenti C, et al. A report from the Brescia Renal COVID Task Force on the clinical characteristics and short-term outcome of hemodialysis patients with SARS-CoV-2 infection. Kidney Int 2020;98:20-6.

8. Mazzoleni L, Ghafari C, Mestrez F, et al. COVID-19 outbreak in a hemodialysis center: a retrospective monocentric case series. Can J Kidney Health Dis 2020;7: 2054358120944298.

9. Corbett RW, Blakey S, Nitsch D, et al. Epidemiology of COVID-19 in an urban dialysis center. J Am Soc Nephrol 2020;31:1815-23.

10. Savino M, Casula A, Santhakumaran S, et al. Sociodemographic features and mortality of individuals on haemodialysis treatment who test positive for SARS-CoV-2: a UK Renal Registry data analysis. PLoS One 2020;15:e0241263.

11. About us. Toronto: Ontario Renal Network. Available: www.ontariorenalnetwork.ca /en/about (accessed 2020 July 13).

12. COVID-19 quick reference public health guidance on testing and clearance. Toronto: Ontario Ministry of Health; 2020. Available: health.gov.on.ca/en/pro/ programs/publichealth/coronavirus/docs/2019_testing_clearing_cases_guidance .pdf (accessed 2020 Aug. 26).

13. Mamdani M, Sykora K, Li P, et al. Reader's guide to critical appraisal of cohort studies: 2. Assessment potential for confounding. BMJ 2005;330:960-2.

14. Ontario Demographic Quarterly: highlights of first quarter 2020. Toronto: Government of Ontario; updated 2020 June 23. Available: www.ontario.ca/page/ontario -demographic-quarterly-highlights-first-quarter-2020 (accessed 2021 Jan. 6).

15. COVID-19 surveillance report for renal centres in the UK: all regions and centres. Bristol (UK): The Renal Association; 2020. Available: https://renal.org/sites/renal. org/files/ALL_REGIONS_CENTRES_covid_report_26082020.pdf (accessed 2020 Aug. 26).
16. Goicoechea M, Cámara LAS, Macías N, et al. COVID-19: clinical course and outcomes of 36 hemodialysis patients in Spain. Kidney Int 2020;98:27-34.

17. Tortonese S, Scriabine I, Anjou L, et al.; AP-HP/Universities/Inserm COVID-19 research collaboration. COVID-19 in patients on maintenance dialysis in the Paris region. Kidney Int Rep 2020;5:1535-44.

18. Lano G, Braconnier A, Bataille S, et al. Risk factors for severity of COVID-19 in chronic dialysis patients from a multicentre French cohort. Clin Kidney J 2020;13:878-88.

19. COVID-19 Task Force Committee of the Japanese Association of Dialysis Physicians; Japanese Society for Dialysis Therapy; Japanese Society of Nephrology; Kikuchi K, Nangaku M, Ryuzaki M, et al. COVID-19 of dialysis patients in Japan: current status and guidance on preventive measures. Ther Apher Dial 2020;24:361-5.

20. Fisher M, Yunes M, Mokrzycki MH, et al. Chronic hemodialysis patients hospitalized with COVID-19: short-term outcomes in the Bronx, New York. Kidney360 2020; 1: 755-62.

21. Bigelow BF, Tang O, Toci GR, et al. Transmission of SARS-CoV-2 involving residents ceceiving dialysis in a nursing home: Maryland, April 2020. MMWR Morb Mortal Wkly Rep 2020;69:1089-94.

22. Brown EA, Perl J. Increasing peritoneal dialysis use in response to the COVID-19 pandemic: Will it go viral? J Am Soc Nephrol 2020;31:1928-30.

23. Socio-demographic data and equity in health services in Ontario: building on strong foundations. Toronto: Wellesley Institute: 2017. Available: www.wellesleyinstitute. com/wp-content/uploads/2017/10/Collecting-Socio-demographic-Data.pdf (accessed 2020 Aug. 26).

24. COVID-19: status of cases in Toronto. Toronto: City of Toronto. Available: www. toronto.ca/home/covid-19/covid-19-latest-city-of-toronto-news/covid-19-status-of -cases-in-toronto/ (accessed 2020 Aug. 26).
Competing interests: Leena Taji, Doneal Thomas, Jane Ip, Yiwen Tang, Angie Yeung and Rebecca Cooper are salaried employees of Ontario Renal Network, Ontario Health. Matthew Oliver, Phil McFarlane and Peter Blake are contracted Medical Leads at Ontario Renal Network, Ontario Health. Matthew Oliver is owner of Oliver Medical Management Inc., which licenses Dialysis Management Analysis and Reporting System software. He has received honoraria for speaking from Baxter Healthcare and participated on Advisory Boards for Janssen and Amgen. Peter Blake has received honoraria from Baxter Global for speaking engagements. Andrew House has received honoraria from Baxter for speaking engagements. Phil McFarlane has received consultant or lecture fees from Amgen, Astra-Zeneca, Bayer, BMS, Boehringer-Ingelheim, GSK, Janssen, Lilly, Novartis, Otsuka, SanofiAventis, Servier and Vifor. He has also received research grants from Amgen, Astra-Zeneca, Bayer, Boehringer-Ingelheim and Otsuka. No other competing interests were declared.

This article has been peer reviewed.

Affiliations: Ontario Renal Network (Taji, Thomas, Oliver, Ip, Tang, Yeung, Cooper, McFarlane, Blake), Ontario Health; Department of Medicine (Oliver), University of Toronto, Toronto, Ont.; Schulich School of Medicine and Dentistry (House, Blake), Western University, London, Ont.; St. Michael's Hospital (McFarlane), Toronto, Ont.

Contributors: Leena Taji wrote the original draft and supervised the collection of the data on SARS-CoV-2 from the Renal Programs. Angie Yeung assisted in writing the initial draft and in coordinating data analysis. Doneal Thomas, Jane Ip and Yiwen Tang did the statistical analysis. Peter Blake revised the manuscript. Matthew Oliver, Phil McFarlane and Andrew House contributed to the conception of the article. Phil McFarlane, Andrew House, Rebecca Cooper and Peter Blake revised the manuscript critically for important intellectual content. All of the authors gave final approval of the version to be published and agreed to be accountable for all aspects of the work.

Content licence: This is an Open Access article distributed in accordance with the terms of the Creative Commons Attribution (CC BY-NC-ND 4.0) licence, which permits use, distribution and reproduction in any medium, provided that the original publication is properly cited, the use is noncommercial (i.e., research or educational use), and no modifications or adaptations are made. See: https://creativecommons.org/licenses/by-nc-nd/4.0/
Funding: The authors received no financial support for the research, authorship and/or publication of this article, other than as employees of Ontario Renal Network.

Data sharing: Ontario Health is prohibited from making the data used in this research publicly accessible if they include potentially identifiable personal health information and/or personal information as defined in Ontario law, specifically the Personal Health Information Protection Act and the Freedom of Information and Protection of Privacy Act. Upon request, data de-identified to a level suitable for public release may be provided.

Acknowledgements: The authors thank the Ontario Regional Renal Programs and all individuals submitting data each week for their efforts to serve those with kidney disease.

Data acknowledgements: The authors acknowledge that data used in this publication were obtained through the Ontario Renal Reporting System and the ORN COVID-19 Data tracker, collected and provided by the Ontario Renal Network, a part of Ontario Health.

Disclaimer: This study was supported by ICES, which is funded by an annual grant from the Ontario Ministry of Health and Long-Term Care (MOHLTC). The opinions, results and conclusions reported in this paper are those of the authors and are independent from the funding sources. No endorsement by ICES or the Ontario MOHLTC is intended or should be inferred.

Parts of this manuscript are based on data and information compiled and provided by the Canadian Institute for Health Information ( $\mathrm{ClHI}$ ). However, the analyses, conclusions, opinions and statements expressed herein are those of the authors, and not necessarily those of $\mathrm{CIHI}$.

Source: Neighbourhood income quintile analysis adapted from Statistics Canada Postal CodeOM Conversion File and/or Postal CodesOM by Federal Ridings File and/or Postal CodeOM Conversion File Plus (November 2018), which is based on data licensed from Canada Post Corporation. Ontario Health has a licence that allows use of this data. It is described in the last paragraph of Data Sources in the Methods section.

Accepted: Jan. 18, 2021

Correspondence to: Peter Blake, peter.blake@lhsc.on.ca 